\title{
Effects of Different Methods of Resistance Training on Indicators of Daily Physical Fitness in Physically Active Elderly Women
}

\author{
Ilmar Danilo Santos Lima ${ }^{1}$, Antonio Gomes de Resende-Neto ${ }^{*}$, Jose Carlos Aragao- \\ Santos ${ }^{2}$, Albernon Costa Nogueira ${ }^{1}$, Alan Bruno Silva Vasconcelos ${ }^{2}$, Bruna Caroline Oliveira \\ Andrade $^{1}$, Iohanna Gilnara Santos Fernandes ${ }^{1}$ and Marzo Edir Da Silva-Grigoletto ${ }^{1,2}$
}

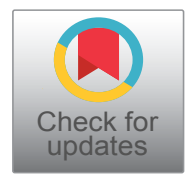

${ }^{1}$ Department of Physical Education, Federal University of Sergipe, Center for Biological and Health Sciences, Brazil ${ }^{2}$ Department of Physiology, Federal University of Sergipe, Center for Biological and Health Sciences, Brazil

* Corresponding author: Antonio Gomes de Resende-Neto, Department of Physical Education, Federal University of Sergipe, Center for Biological and Health Sciences, Rua Projetada III, 189, BL12 AP304, Bairro Rosa Elze, CEP: 49100-00, Sao Cristovao- SE, Brazil

\begin{abstract}
Aim: To compare changes after 12 weeks of functional and traditional training in physical fitness related to daily activities in physically active elderly women.

Methods: 30 elderly women were randomized into two groups: 1) Functional Training (FT $-n=15,65.12 \pm 4.49$ years) and 2) and Traditional Training (TT - $n=15,64.87$ \pm 3.25 years). For the verification of functional responses, the following tests were applied: Dress and Undress a Sleeveless Shirt (DUSS), Gallon-Jug Shelf-Transfer (GJSF), Get up from the chair and move around the house (GCMA), Sit and Stand up in 5 Reps (SS5R) and 400 meters walk. Data were analyzed from a repeated measures ANOVA followed by Bonferroni's post-hoc.

Results: After 12 weeks, both FT and TT showed significant increases in the power of lower limbs (SS5R: FT + 18.0\%, TT $+21.7 \%$ ) compared to the initial values. The FT showed statistically significant differences in agility/dynamic balance (GCMA: $+5.3 \%, p=0.02$ ) and cardiorespiratory capacity (400 W: + 10.4\%; $p=0.007$ ); and TT showed significant improvements in mobility and overall coordination (GJSF: $+8.1 \%, p=0.001)$ when compared to the pre-test. No differences were found between the groups in any of the analyzes.

Conclusion: The training protocols analyzed are equally effective for the improvement of indicators of physical fitness in physically active elderly women and can be alternately administered in health promotion programs.
\end{abstract}

\section{Keywords}

Aging, Physical exercise, Daily activities

\section{Introduction}

Aging is an intrinsic process of the human being linked to functional deteriorations in several systems, affecting the quality of life due to the inability to perform the simple tasks of the day to day in an autonomous, efficient and safe way [1]. In addition, the female sex is also considered an independent risk factor for functional capacity decline. When compared to men, women are more affected by senescence due to hormonal changes associated with menopause that intensify muscle weakness and increases the risk of mortality [2].

In this context, physical exercise, especially strength training, appears as a countermeasure to attenuate these negative modifications, being a non-pharmacological strategy considered efficient in promoting adaptations in the physical fitness and health of those who perform [3].

Functional (FT) and Traditional (TT) Training are presented as effective methods in promoting multisystem adaptations in the elderly [4]. The TT is more analytical, uniarticular and uniplanar performing a more isolated work, usually in bodybuilding machines and very linked to aesthetic purposes, has its proven influence in relation to functional and structural adaptations $[5,6]$, but there are questions about its effects on performance improvement in daily activities of elderly [7]. On the

Citation: Lima IDS, Resende-Neto AG, Aragao-Santos JC, Nogueira AC, Vasconcelos ABS, et al. (2019) Effects of Different Methods of Resistance Training on Indicators of Daily Physical Fitness in Physically Active Elderly Women. Int J Sports Exerc Med 5:136. doi.org/10.23937/2469-5718/1510136 Accepted: July 27, 2019; Published: July 29, 2019

Copyright: (c) 2019 Lima IDS, et al. This is an open-access article distributed under the terms of the Creative Commons Attribution License, which permits unrestricted use, distribution, and reproduction in any medium, provided the original author and source are credited. 
Table 1: Characteristics of participants in functional (FT) and traditional (TT) training. Values presented in mean and standard deviation $(M \pm S D)$.

\begin{tabular}{|l|l|l|l|}
\hline Variables & FT $(\mathbf{n}=\mathbf{1 5})$ & TT $(\mathbf{n}=\mathbf{1 5})$ & $\boldsymbol{p}$ \\
\hline Age & $65.12 \pm 4.49$ & $64.87 \pm 3.25$ & 0.94 \\
\hline BMl, $\mathrm{kg} / \mathrm{m}^{2}$ & $29.09 \pm 4.81$ & $27.51 \pm 4.84$ & 0.37 \\
\hline Waist-to-hip ratio, cm & $0.85 \pm 0.09$ & $0.88 \pm 0.07$ & 0.25 \\
\hline
\end{tabular}

Note: No significant difference $(p<0.05)$ was found for any of the variables analyzed in the pre-test.

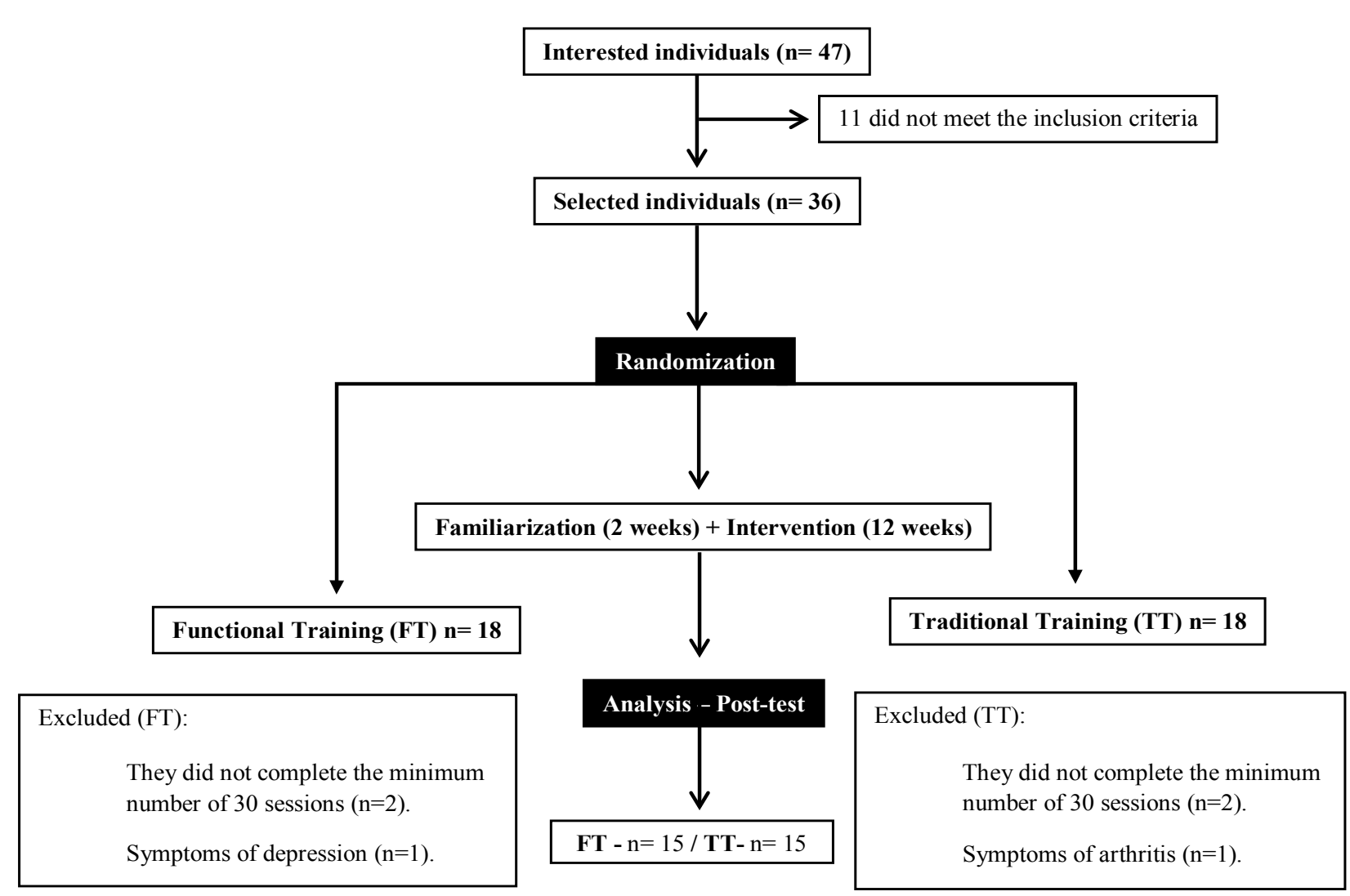

Figure 1: Experimental design of the study.

other hand, FT has been much used, since several physical capacities are stimulated in a single training session, recommending the use of multi segmental and multiplanar exercises, combined with acceleration and deceleration, and stabilization of movements, aiming the transfer of the results obtained for daily activities [8].

However, there is also a lack of a systematized FT protocol in the scientific literature, as well as the lack of investigations comparing FT with traditional training methods, which hinders comparison between the protocols used and between the responses found $[9,10]$. Thus, the objective of the present study was to compare the changes after 12 weeks of functional and traditional training in physical fitness related to daily activities in physically active elderly women. The differential in relation to previous works is the methodological rigor and a clear standardization of the intervention protocols. Our hypothesis is that specific training protocols for activities of daily life, which require greater activation of stabilizing muscles, can promote more effective adaptive responses in the functionality of older women.

\section{Methods}

It is a study with an experimental design to apply specific treatments in groups of individuals, aiming to control the intervening factors and to describe the behavior of the variables observed from an intervention. This study was approved by the Ethics Committee of the Federal University of Sergipe (No. 3.087.488) and followed all the ethical aspects of the Declaration of Helsinki.

\section{Subjects}

Thirty physically active elderly women were randomly assigned to two different groups: functional training $\left(\mathrm{FT}-\mathrm{n}=15 ; 29.09 \pm 4.81 \mathrm{~kg} / \mathrm{m}^{2}\right)$ and traditional training $\left(\mathrm{TT}-\mathrm{n}=15 ; 27.51 \pm 4.84 \mathrm{~kg} / \mathrm{m}^{2}\right)$. All participants underwent a medical visit in order to prove the suitability for the activities of the intervention, and physical and nutritional assessment. Inclusion criteria were: age above 60 years; women; practitioners of regular physical exercise in the last three months and free of musculoskeletal or cardiovascular condition that would contraindicate the performance of the training. 
All participants, whose characteristics are presented in Table 1, lived at home, had assistance from the public health system and carried out common actions, such as walking and domestic activities, independently (Figure 1).

\section{Exercise programs}

The intervention period was 12 weeks, in which three weekly training sessions were held, each lasting 50 minutes on non-consecutive days, respecting the recovery time of 48 hours. The OMNI-GSE scale [11] was used to control and normalize the overall internal load of the different protocols, from which the participants were asked to choose a score that reflected their perceived effort after each exercise block.

After the initial measurements, all the volunteers went through two weeks of familiarization with the methods in which $50 \%$ of the intensity planned for the first session was applied, and finally completed 36 training sessions. Each session was divided into four blocks, where during blocks 1 and 4 the groups performed the same activities together.

Thus, both experimental groups performed $10 \mathrm{~min}$ utes of joint mobility exercises and five final minutes of intermittent activities. After block 1, the FT group performed 15 minutes of intermittent activities (Block 2), organized in a circuit format that required the components of agility, motor coordination, speed and muscular power. At the end of this block, two minutes of rest were allowed to initiate block 3 , in which 20 minutes of resistance exercises performed at maximum concentric velocity were performed, with strengthening stimulus for all regions of the body, organized in a circuit format for lower limbs, upper limbs and body middle zone.

During block 2 of the TT, a 15-minute interval walk
(30 seconds walking naturally alternating with $30 \mathrm{sec}-$ onds walking a little faster) was performed within a distance of 86 meters. At the end TT participants rested for two minutes and then started block 3 , in which they performed for 20 minutes traditional strength exercises on bodybuilders with isolated neuromuscular work, performed at maximum concentric speed, in a circuit format, alternating the body segments (Figure 2).

For the TT group, the addition of external load during the training period occurred from a perceived effort 6 (easy) on the OMINI-GSE scale and with maintenance of 08 to 12 maximum repetitions. For the FT group, the same criterion was followed for addition of external load in the possible exercises and exercises performed with the body mass itself, biomechanical modifications were applied according to the level of comfort and ability of the individual, for maintenance of 08 to 12 repetitions.

The present intervention proposal was elaborated according to the concepts presented by La Scala Teixeira, et al. [8] and was previously tested by Resende-Neto, et al. [12].

\section{Collection procedures}

The tests were performed at the same time and place, administered in the same order, supervised by the same researchers before and after the 12-week intervention. Before running the tests, the researchers adjusted the equipment and instructed the participants about body positioning. All participants wore sports clothing and were instructed to give the maximum effort during the tests, being motivated by means of clapping and incentive phrases.

For the anthropometric characterization the body mass $(\mathrm{kg})$ was determined through a scale $\left(\right.$ Lider $^{\circledR}$,

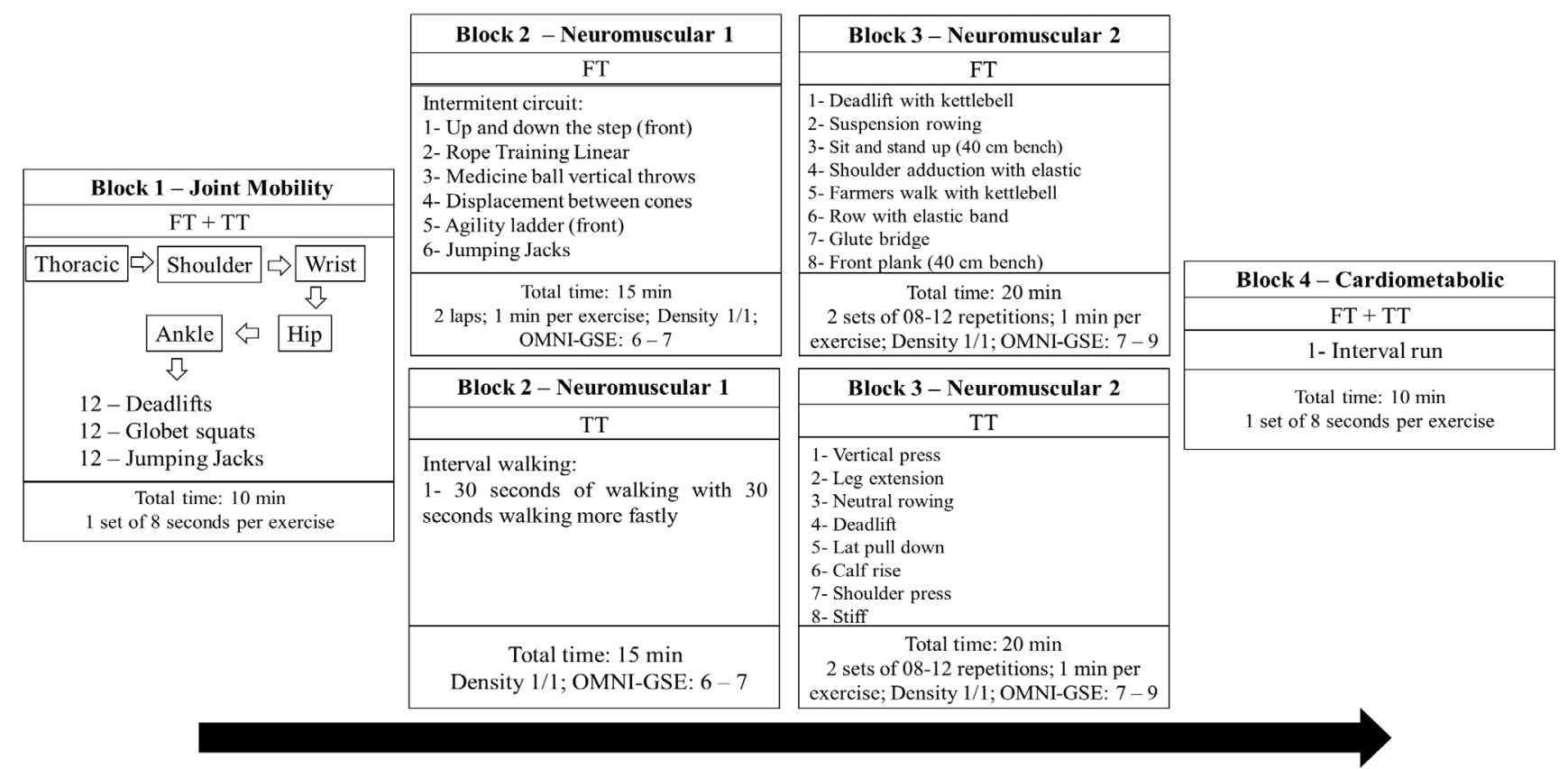

Figure 2: Overview of functional training sessions (FT) and traditional strength training (TT). 
P150C, Sao Paulo, Brazil), with a maximum capacity of $150 \mathrm{~kg}$. Stature $(\mathrm{cm})$ was determined using a stadiometer (Sanny, ES2030, Sao Paulo, Brazil). The Body Mass Index (BMI) was obtained using the formula $\mathrm{kg} / \mathrm{m}^{2}$. With an anthropometric tape, the waist and hip circumference were determined to obtain the Waist-o-Hip Ratio (WHR).

For the verification of the functional aptitude related to daily activities, the following tests were used:

a) Dress and Undress a Sleeveless Shirt (DUSS): aims to evaluate the mobility of the glenohumeral joint and coordination of upper limbs. The individual was instructed to stand with arms over his body and with a T-shirt in one hand. At the "now" sign, she donned the T-shirt and immediately undressed, returning to the starting position. The shortest time of three attempts was recorded [13].

b) Gallon-Jug Shelf-Transfer (GJST): aims to evaluate coordination and global mobility. The participants were instructed to transfer in sequence five bottles from a lower shelf to an upper one located at the level of the participant's shoulder. Before the test, the participants were instructed regarding the safety, moving one bottle at a time and without alternating the use of the hand. The time was started in the "go" command and finalized when the last gallon was transferred to the upper shelf, two attempts were made and the shortest time was recorded [14].

c) Get up from the Chair and Move around the house (GCMA): aims to evaluate agility and dynamic balance. The test began with the participant sitting in a chair, hands on the thighs and feet resting on the ground. At the sign of the evaluator, the participant raised and moved to the right, circled the cone, returned to the chair, sat down, and withdrew both feet off the floor. Without hesitation, she stood up again, moved to the left, circled the cone, and sat down again, pulling both feet off the ground. Immediately, he made a new circuit (exactly the same as the first one). Thus, the course consisted of circumventing each cone twice, alternately to the right, to the left, to the right and to the left. Two attempts were made and the shorter time was recorded [15].

d) Sit and Stand up in 5 Reps (SS5R): to evaluate the potency of the lower limbs. This test is intended to evaluate an individual's ability to sit and stand up from the chair as quickly as possible. The time it takes to sit and lift the chair in five repetitions without the use of the arms will be timed. Three attempts were made and the shorter time was recorded [16].

e) 400 - meter walk: to assess cardiovascular fitness. Participants were instructed to complete a lap of 400 meters as fast as possible, the time they used to make the lap was timed in seconds and converted to m/s [17].

\section{Statistical analysis}

The sample size was calculated using G*Power software version 3.1.9.2 based on previous results [18]. In the GJST test we expected a change of about $1.5 \mathrm{sec}$ onds in the recorded time, being the main variable to verify the functionality in the present study, thus, we consider for the sample of the present study a power of 0,85 for the analyzes performed.

Descriptive analysis with mean, standard deviation and percentage delta was used to characterize all variables obtained. The homogeneity of the data was confirmed from the Levene test. ANOVA $(2 \times 2)$ followed by Bonferroni post hoc was used to verify the differences between the groups. Tabulated and analyzed using the Statistical Package for Social Sciences (SPSS), version 22, adopting a significance level of $5 \%(p \leq 0.05)$. The Effect Size (ES) was calculated according to the methodological procedures defined by Cohen [19].

\section{Results}

The average participation rate was 33 sessions of 36 and the sample loss was six participants. The FT obtained three losses, one by medical waiver and two for failure to comply with all stages of the study. TT also obtained three losses, one by medical waiver and two for less than $85 \%$ of training sessions.

After 12 weeks, both FT and TT showed significant increases in the muscle power of lower limbs (Sit and stand up in 5 reps) compared to the initial values. The FT showed statistically significant differences in agility/ dynamic balance $(p=0.02)$ and in cardiovascular fitness ( $p=0.007)$; and TT showed significant improvements in mobility and overall coordination $(p=0.001)$ when compared to the pre-test. No differences were found between the groups in any of the analyzes (Figure 3).

\section{Discussion}

The present study compared the effects of 12 weeks of functional training and traditional training on physical fitness for daily activities of the elderly. The main finding was that both methods promoted similar responses in the analyzed components.

The mobility of glenohumeral and coordination of upper limbs did not present statistical difference between the groups, however, there was a percentage improvement. This adaptation is possibly explained by the mobility exercises applied in the first training block. In this perspective, Cunha, et al. [20] obtained significant improvements in this same test from a walking protocol together with a previous warm-up with joint exercises.

Coordination and global mobility were only modulated by TT. Corroborating the present findings, Balachandran, et al. [18] after a 12-week intervention in machine strength training, found a $15 \%$ increase in the GJST test in independent older adults. Possibly, the precise in- 

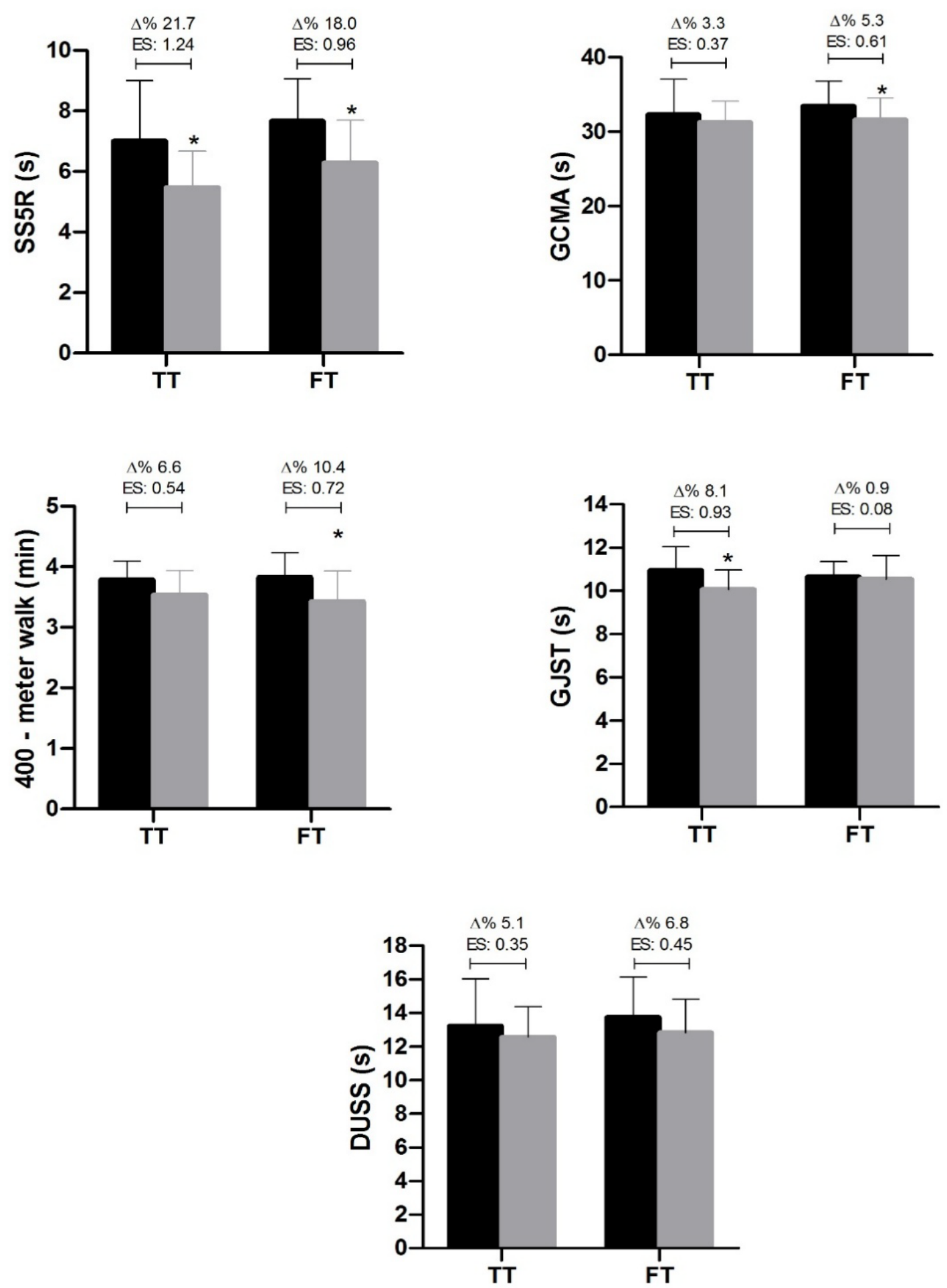

Figure 3: Changes after 12 weeks of functional (FT) and traditional (TT) training in physical fitness related to daily activities. Values presented in mean and standard deviation $(M \pm S D)$.

Note: "Significant statistical difference pre vs. post intervention $(p \leq 0.05) . \Delta \%$ - Delta Percentage between pre and posttest. ES - Effect Size. DUSS - Dress and Undress a Sleeveless Shirt. GJSF - Gallon-Jug Shelf-Transfer. GCMA - Get up from the Chair and move around the house. SS5R - Sit and Stand up in 5 Reps.

crease of the physical efforts during the training is associated to the greater control of the load. The exercises applied in the TT were mostly multiarcular and focused on functional pull and push actions, which are essential for the good performance of the GJST.

Agility and dynamic balance were sensitive to the stimulus proposed by FT. The reduction in time is cer- tainly related to the specificity of the training that was composed by high-speed displacements and jumps that emphasize muscular power. On the other hand, block 2 of the TT performed an unspecific walk. The findings of the present study confirm together with Liu, et al. [9], that the effects of FT are proportionally dependent on the tasks performed during the training itself. 
The power of the lower limbs showed positive results for both groups. Among the possible explanations, we believe that there has been an improvement in neuromuscular coordination and increases in the area of fast-twitch muscle fibers prompted by the high intensity and speed of the applied training. Confirmatively, Fleck and Kraemer [21] identified a significant increase in the area of type II fibers, not significant in type I fibers, through biopsies in muscles trained in high intensity force.

Cardiorespiratory capacity was significantly modified in FT, but TT also showed a clinically considerable improvement. This decrease in test run time is possibly related to the training trait of encompassing various components of physical fitness such as strength and aerobic endurance. Bouaziz, et al. [4] showed that multicomponent training positively affects both functional ability performance and cardiorespiratory fitness in the elderly. In addition, studies have observed increases in the cardiorespiratory capacity of elderly subjects who underwent strength training $[22,23]$.

The present study exposes two safe and effective methods, of easy reproducibility and practical application that can help to counterbalance the reductions in the neuromuscular system caused by the advancing age. However, some limitations must be taken into account when interpreting the results. First, losses throughout the study, however the statistical power was reached and, consequently, did not affect the results obtained. Furthermore, the participants in our study were older women physically able to perform a physical training program of high volume and intensity and may not be fully representative of the general population of the elderly. Future studies should perform longer interventions with a larger sample, verify functional responses with more scientifically validated tools, as well as analyze more variables, such as body composition and inflammatory markers, in order to ascertain other conditions associated with aging.

\section{Conclusion}

The functional and traditional training were efficient in improving physical fitness related to the daily activities of physically active elderly women. The present study shows that both protocols with specific exercises as well as general exercises result in similar improvements in the functionality of elderly individuals. Thus, this work offers new alternatives for professionals in the field and can contribute to a better efficiency of health promotion programs through physical exercises.

\section{Conflict of Interest}

The authors declare no conflict of interest with the contents of this article.

\section{Author Contributions}

IDSL, AGRN and ACN designed research; IDSL, IGSF and ABSV performed experiments; AGRN and MESG analyzed data; AGRN and JCAS interpreted results of experiments; IDSL, AGRN and JCAS drafted manuscript; AGRN and ESC edited and revised manuscript; AGRN and MESG approved final version of manuscript.

\section{Acknowledgments}

No external financial support.

\section{References}

1. Hunter SK, Pereira HM, Keenan KG (2016) The aging neuromuscular system and motor performance. J Appl Physiol (1985) 121: 982-995.

2. Straight CR, Brady AO, Evans E (2015) Sex-specific relationships of physical activity, body composition, and muscle quality with lower-extremity physical function in older men and women. Menopause 22: 297-303.

3. Daskalopoulou C, Stubbs B, Kralj C, Koukounari A, Prince $M$, et al. (2017) Physical activity and healthy ageing: $A$ systematic review and meta-analysis of longitudinal cohort studies. Ageing Res Rev 38: 6-17.

4. Bouaziz W, Lang PO, Schmitt E, Kaltenbach G, Geny B, et al. (2016) Health benefits of multicomponent training programmes in seniors: a systematic review. Int J Clin Pract 70: $520-536$.

5. Pina FLC, Nunes JP, Nascimento MA, Ribeiro AS, Mayhew $\mathrm{JL}$, et al. (2019) Similar Effects of 24 Weeks of Resistance Training Performed with Different Frequencies on Muscle Strength, Muscle Mass, and Muscle Quality in Older Women. Int J Exerc Sci 12: 623-635.

6. Marcos-Pardo PJ, Orquin-Castrillón FJ, Gea-García GM, Menayo-Antúnez R, González-Gálvez N, et al. (2019) Effects of a moderate-to-high intensity resistance circuit training on fat mass, functional capacity, muscular strength, and quality of life in elderly: A randomized controlled trial. Sci Rep 9: 7830.

7. Latham NK, Bennett DA, Stretton CM, Anderson CS (2004) Systematic review of progressive resistance strength training in older adults. J Gerontol A Biol Sci Med Sci 59: 48-61.

8. La Scala Teixeira CV, Evangelista AL, Novaes JS, Da Silva Grigoletto ME, Behm DG (2017) "You're Only as Strong as Your Weakest Link": A Current Opinion about the Concepts and Characteristics of Functional Training. Front Physiol 8: 643.

9. Liu C, Shiroy DM, Jones LY, Clark DO (2014) Systematic review of functional training on muscle strength, physical functioning, and activities of daily living in older adults. Eur Rev Aging Phys Act 11: 95-106.

10. Marín-Cascales E, Alcaraz PE, Ramos-Campo DJ, RubioArias JA (2017) Effects of multicomponent training on lean and bone mass in postmenopausal and older women: a systematic review. Menopause 25: 346-356.

11. Da Silva-Grigoletto $\mathrm{M}$, Viana-Montaner $\mathrm{BH}$, Heredia JR, Mata F (2013) Validación de la escala de valoración subjetiva del esfuerzo OMNI-GSE para el control de la intensidad global en sesiones de objetivos múltiples en personas mayores.

12. Resende-Neto AG, Santos MS, Silva RJS, De Santana JM, Da Silva-Grigoletto ME (2018) Effects of different neuromuscular training protocols on the functional capacity of elderly women. Rev Bras Med Esporte 24: 140-144.

13. Martin Dantas EH, Gomes de Souza Vale R (2004) 
Protocolo GDLAM de avaliaçao da autonomia funcional. Fit Perform J 3: 175-183.

14. Signorile JF, Sandler D, Ma F, Bamel S, Stanziano D, et al (2007) The gallon-jug shelf-transfer test: an instrument to evaluate deteriorating function in older adults. J Aging Phys Act 15: 56-74.

15. Andreotti RA, Silene Sumire Okuma (2017) Validação de uma bateria de testes de atividades da vida diária para idosos fisicamente independentes. Rev Paul Educ Física 13: $46-66$.

16. Pereira A, Izquierdo M, Silva AJ, Costa AM, Bastos E, et al. (2012) Effects of high-speed power training on functional capacity and muscle performance in older women. Exp Gerontol 47: 250-255.

17. Simonsick EM, Montgomery PS, Newman AB, Bauer DC, Harris T (2001) Measuring fitness in healthy older adults: the Health $A B C$ Long Distance Corridor Walk. J Am Geriatr Soc 49: 1544-1548.

18. Balachandran A, Martins MM, De Faveri FG, Alan O
Cetinkaya F, et al. (2016) Functional strength training: Seated machine vs standing cable training to improve physical function in elderly. Exp Gerontol 82: 131-138.

19. Cohen J (1988) Statistical power analysis for the behavioral sciences. ( $2^{\text {nd }}$ edn), L. Erlbaum Associates, Hillsdale NJ.

20. Cunha RCL da, Fortes M de SR, Ferreira M de A (2010) Efeitos de um programa de caminhada sob os níveis de autonomia funcional de idosas monitoradas pelo programa saúde da família. Rev Bras Geriatr E Gerontol 13: 255-265.

21. Fleck SJ, Kraemer WJ, Ribeiro JL (2006) Fundamentos do treinamento de força muscular. ArtMed, Porto Alegre.

22. Frontera WR, Meredith CN, O'Reilly KP, Evans WJ (1990) Strength training and determinants of VO2max in older men. J Appl Physiol (1985) 68: 329-333.

23. Vincent KR, Braith RW, Feldman RA, Magyari PM, Cutler $\mathrm{RB}$, et al. (2002) Resistance exercise and physical performance in adults aged 60 to 83. J Am Geriatr Soc 50: 11001107. 